\title{
An Effective Beamformer for Coherent Signal Reception
}

\author{
Linrang Zhang', H.C. So ${ }^{2}$, Li Ping ${ }^{3}$ and Guisheng Liao ${ }^{1}$ \\ ${ }^{1}$ National Key Laboratory of Radar Signal Processing \\ Xidian University, Xi'an, China \\ ${ }^{2}$ Department of Computer Engineering \& Information Technology \\ City University of Hong Kong, Kowloon, Hong Kong \\ ${ }^{3}$ Department of Electronic Engineering \\ City University of Hong Kong, Kowloon, Hong Kong
}

\begin{abstract}
This letter proposes a new two-stage beamformer for coherent signal reception. The first stage extracts all the coherent signals from an antenna array with the use of their direction-of-arrival information, and then the resultant signals are coherently combined in the second stage. Simulation results are included to demonstrate the effectiveness of the proposed technique.
\end{abstract}

Keywords: Beamforming; Coherent signals; Array processing

\section{Introduction}

It is well known [1] that the conventional optimum beamformer, which assumes a single signal source, fails to perform optimally in the presence of multiple coherent sources. Typical approaches for coherent signal reception are to use averaging techniques in the spatial or frequency domains to destroy the coherent components prior to beamforming. After de-correlating the coherent signals, the beamformer can put nulls in their directions-of-arrival (DOAs). 
However, in many scenarios such as multipath propagation, steering the nulls in the directions of the coherent signals is not desirable. It is because in order to fully utilize the information of the coherent signals, these signals should be constructively combined instead of canceling all but one of them. A number of blind beamformers, which make use of the desired signal properties, such as the cumulant [2] and cyclostationary [3] approaches, have been proposed to achieve better performance. However, these methods are generally difficult to realize and need a large number of snapshots for convergence. A multiple constrained method has also been suggested in [4] by using high-order null constraints in the DOAs associated with the coherent signals in order to prevent signal cancellation. Since this algorithm does not exploit the energy of all coherent signals,

optimum performance cannot be achieved. In this Letter, an improvement to [4] for efficient coherent signal reception is devised.

\section{Problem Formulation}

Assume that plane waves emitted by a desired group of $P$ coherent sources and $J$ uncorrelated interferences impinge on a $M$-element antenna array from directions $\theta_{d i}$, $i=1,2, \ldots, P$ and $\theta_{u i}, i=1,2, \ldots, J$, respectively. These sources are assumed to be narrowband with the same center frequency and in the far field of the array. The received array signal vector can be expressed as

$$
\mathbf{x}(t)=s_{d}(t) \sum_{i=1}^{P} \alpha_{i} \mathbf{a}\left(\theta_{d i}\right)+\sum_{i=1}^{J} s_{i}(t) \mathbf{a}\left(\theta_{u i}\right)+\mathbf{n}(t),
$$

where $s_{d}(t)$ represents the desired signal waveform, and $s_{i}(t)$ represents the $i$ th interference waveform, $i=1,2, \ldots, J$. The quantity $\alpha_{i}$ represents the unknown complex amplitude of the $i$ th coherent signal, the $M \times 1$ vector $\mathbf{a}(\theta)$ is called the steering vector of the array, and $\mathbf{n}(t)$ is the spatially white noise vector. It is assumed that $s_{d}(t),\left\{s_{i}(t)\right\}$, and $\mathbf{n}(t)$ are uncorrelated with each other, and the DOA information of the coherent signals is available or has already been accurately estimated [1]. Denote the composite vector associated with the coherent sources as

$$
\widetilde{\mathbf{a}}=\sum_{i=1}^{P} \alpha_{i} \mathbf{a}\left(\theta_{d i}\right)=\mathbf{A} \cdot \boldsymbol{\alpha}
$$


where $\mathbf{A}=\left[\mathbf{a}\left(\theta_{d 1}\right), \mathbf{a}\left(\theta_{d 2}\right), \cdots, \mathbf{a}\left(\theta_{d P}\right)\right]$ and $\boldsymbol{\alpha}=\left[\alpha_{1}, \alpha_{2}, \cdots, \alpha_{P}\right]^{T}$, which is also referred to as the generalized array manifold [2] of the desired signal. Given $\mathbf{x}(t)$ and $\mathbf{A}$, the task is to devise an efficient beamforming weight vector.

\section{New Beamformer Development}

If the waveform of the desired signal is known, that is, when training signal is available, the optimum weight vector $\mathbf{W}$ for processing $\mathbf{x}(t)$ is computed from minimizing the mean square error function $E\left\{\left|s d(t)-\mathbf{W}^{H} \cdot \mathbf{x}(t)\right|^{2}\right\}, \quad$ where $(.)^{H}$ denotes conjugate transposition. The minimum mean square error (MMSE) solution is given by

$$
\mathbf{W}_{M M S E}=\mathbf{R}_{x x}^{-1} \mathbf{r}_{x s}
$$

where $\mathbf{r}_{x s}=E\left\{\mathbf{x}(t) \cdot s d^{*}(t)\right\},(.)^{*}$ is the conjugate operation, and $\mathbf{R} x x=E\left\{\mathbf{x}(t) \mathbf{x}(t)^{H}\right\}$ is the correlation matrix of the array vector. In the MMSE beamformer, the multiple correlated wavefronts corresponding to the coherent signals are optimally combined to maximize the output signal-to-noise ratio (SNR). However, using training signal implies a waste of bandwidth and it may not be available in many practical situations. In the following, a new blind beamformer, which requires no training signals, is developed.

We first use the DOA information of the coherent signals to construct multiple constraints for the minimum variance beamformer, such that the coherent signals are preserved and signal cancellation is prevented. This multiple constrained minimum variance (MCMV) beamformer is determined from the following constrained optimization problem [4]

$$
\mathbf{W}_{M C M V}=\arg \min _{\mathbf{W}} E\left\{\left|\mathbf{W}^{H} \cdot \mathbf{x}(t)\right|^{2}\right\}, \quad \text { subject to } \mathbf{A}^{H} \mathbf{W}=\mathbf{f},
$$

where $\mathbf{f}$ is the $p \times 1$ unknown response vector to be determined. It is clear that $\mathbf{f}$ is dependent on $\boldsymbol{\alpha}$. According to adaptive array theory, the optimal weight vector for (4) is given by

$$
\mathbf{W}_{M C M V}=\mathbf{R} x x^{-1} \mathbf{A}\left(\mathbf{A}^{H} \mathbf{R} x x^{-1} \mathbf{A}\right)^{-1} \mathbf{f}
$$

This paper is a postprint of a paper submitted to and accepted for publication in Electronics Letters and is subject to Institution of Engineering and Technology Copyright.

The copy of record is available at IET Digital Library. 
Note that the choice of the constrained vector $\mathbf{f}$ has a significant effect on the system performance. In [4], $\mathbf{f}$ is chosen as $[1,0, \ldots, 0]^{T}$, which corresponds to preserving the signal in only one desired direction and forcing the receiver response to the direction of the remaining $(P-1)$ coherent signals to zero. Although the additional constraints prevent signal cancellation due to coherent signals, this method does not make use of the energy in the coherent signal components and, therefore, is not optimal. In $[5], \mathbf{f}=[1,1, \ldots, 1]$ is suggested to recover all coherent signals. However, this choice of $\mathbf{f}$ can increase the noise energy, which results in output signal-to-interference-plus-noise ratio (SINR) reduction. Although the constrained vector $\mathbf{f}$ can be obtained using some optimization methods, extensive computation is normally involved and this will be prohibitive particularly for real-time applications.

Our idea is to use $P$ constrained vectors that generalize [4], so that all coherent signals can be extracted one by one from each different direction without causing signal cancellation. After all the coherent signals have been extracted, we can then combine them optimally. As a result, the proposed beamforming approach consists of two stages: first use a set of adaptive beamformers to extract the desired signal along each DOA while suppressing all interferences, then constructively combine the extracted signals to achieve nearly optimum signal estimation. The operating procedure of the proposed blind coherent signal receiver can be summarized in the following two steps:

Step1: Extract the desired signal along each of the DOAs using the generalized MCMV beamformer. The adaptive beamforming weight for the $l$ th direction of the desired signal is computed from

$$
\hat{\mathbf{W}}_{l}=\arg \min _{\mathbf{W}_{l}} E\left\{\left|\mathbf{W}_{l}^{H} \cdot \mathbf{x}(t)\right|^{2}\right\}, \text { subject to } \mathbf{A}^{H} \mathbf{W}_{l}=[0 \cdots 1 \cdots 0]^{T} \stackrel{\text { def }}{=} \mathbf{1}_{l}, \quad l=1,2, \ldots, P
$$

where $\mathbf{1}_{l}$ is a vector with all elements equal 0 except 1 at the $l$ th position. The output of the $l$ th filter is given by

$$
\begin{aligned}
y_{l}(t) & =\hat{\mathbf{W}}_{l}^{H} \cdot \mathbf{x}(t)=\hat{\mathbf{W}}_{l}^{H} \cdot s d(t) \sum_{i=1}^{P} \alpha \mathbf{a}\left(\theta_{d i}\right)+\hat{\mathbf{W}}_{l}^{H} \cdot\left[\sum_{i=1}^{J} s_{i}(t) \mathbf{a}\left(\theta_{u i}\right)+\mathbf{n}(t)\right] \\
& =\alpha_{l} s d(t)+e_{l}(t)
\end{aligned}
$$

This paper is a postprint of a paper submitted to and accepted for publication in Electronics Letters and is subject to Institution of Engineering and Technology Copyright.

The copy of record is available at IET Digital Library. 
where $e_{l}(t)$ denotes the residual noise plus interference after filtering. Clearly, $\boldsymbol{W}_{l}$ picks up the desired signal along $\mathbf{a}\left(\theta_{d l}\right)$ without considering the signals along $\mathbf{a}\left(\theta_{d 1}\right), \ldots, \mathbf{a}\left(\theta_{d(l-1)}\right), \mathbf{a}\left(\theta_{d(l+1)}\right), \ldots, \mathbf{a}\left(\theta_{d P}\right)$. As a result, the use of $P$ weight vectors allows us to extract all coherent signals at different directions.

Step2: Coherently combine the first stage filter outputs to increase the SNR of the final signal estimate. Stacking the filter outputs from all coherent signal directions, $\left\{y_{l}(t)\right\}_{l=1}^{P}$, yields a more compact vector form:

$$
\mathbf{y}(t)=\left[\begin{array}{c}
y_{1}(t) \\
y_{2}(t) \\
\vdots \\
y_{P}(t)
\end{array}\right]=\boldsymbol{\alpha} \cdot s_{d}(t)+\mathbf{e}(t) .
$$

Because of interference and noise suppression at the first stage, the total signal power in $\mathbf{y}(\mathrm{t})$ will be significantly higher than that of $\mathbf{e}(\mathrm{t})$. In this case, $\mathbf{R}_{y y}=E\left[\mathbf{y}(t) \cdot \mathbf{y}(t)^{H}\right]=p_{s} \boldsymbol{\alpha} \cdot \boldsymbol{\alpha}^{H}+\mathbf{R}_{e e} \approx p_{s} \boldsymbol{\alpha} \cdot \boldsymbol{\alpha}^{H}$, where $p_{s}$ is the desired signal power. It enables us to approximate the optimum vector using the principal eigenvector of $\mathbf{R}_{\mathrm{yy}}$, which can be easily obtained using standard decomposition techniques, although the approximation is a tradeoff between optimality and complexity. It is noteworthy that the same technique has been employed in practical RAKE receivers in communication systems.

\section{Simulation Results}

Several simulation examples are presented for illustration and comparison. We consider a uniformly linear array with 16 sensors, which corresponds to $M=16$, and the inter-element spacing is equal to the half of the wavelength of the signals. There are four signals impinging on the array from angles of $-40^{\circ},-20^{\circ}, 10^{\circ}$ and $30^{\circ}$, off the array broadside. The first three signals are assumed to be the desired coherent signals with known DOAs but different unknown gains and the fourth signal is an uncorrelated interference with interference-to-noise ratio of $60 \mathrm{~dB}$. 
Example 1: Figure 1 shows the beam patterns of the MMSE and proposed beamformers when the coherent signals have SNRs of $23 \mathrm{~dB}, 25 \mathrm{~dB}$, and $20 \mathrm{~dB}$, respectively. We see that the two-stage beamformer can cope with the problem of coherent signal situation, which produces three main beams with gains proportional to the amplitude of the each coherent signal for simultaneous reception of the desired signals, and a null for successful suppression of the interference. Moreover, its performance is comparable with that of the MMSE approach.

Example 2: This test evaluates the beamformer output SINR as a function of input SNR and the results are plotted in Figure 2. We assume that the SNRs of the three coherent signals are $[-\beta, 0, \beta] \mathrm{dB}$, and the value of $\beta$ is varied from 0 to 20 . Apart from the MMSE and proposed methods, we also include the performance of the MCMV approach [5] with fixed constraints based on $\mathbf{f}=[1,1, \ldots, 1]$. It is observed that the proposed method is superior to the fixed MCMV beamformer particularly when the SNR difference among the three coherent signals is large and its performance approaches that of the MMSE algorithm for the whole range of SNRs.

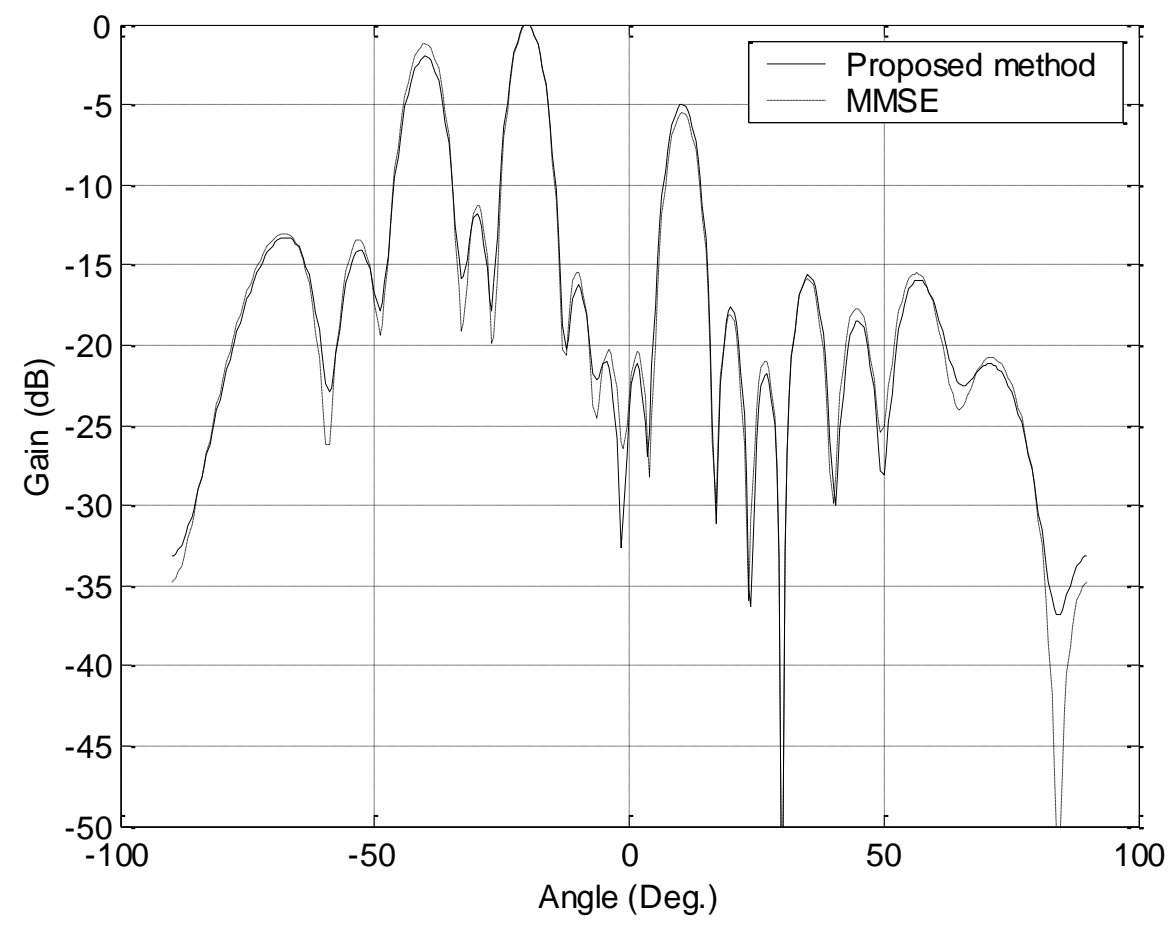

Figure1: Beam pattern

This paper is a postprint of a paper submitted to and accepted for publication in Electronics Letters and is subject to Institution of Engineering and Technology Copyright.

The copy of record is available at IET Digital Library. 


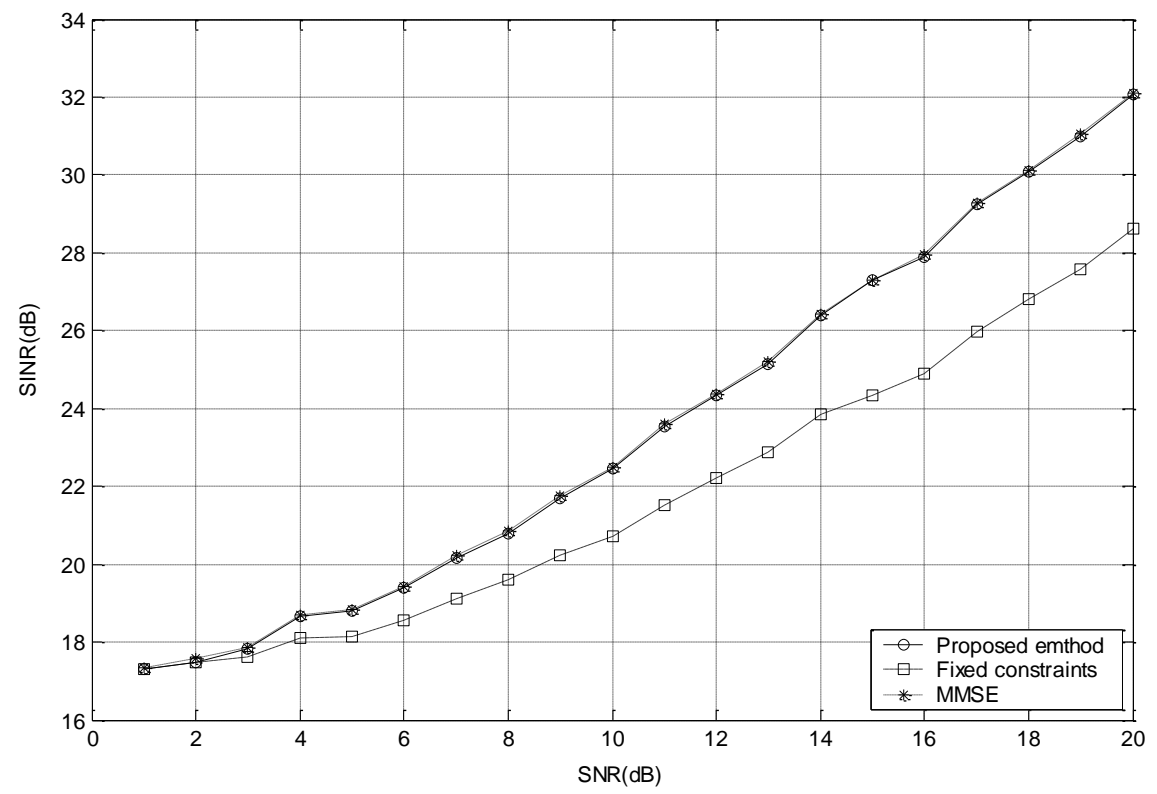

Figure 2: Output SINR versus SNR

\section{Concluding Remarks}

In this Letter, a two-stage beamformer for coherent signal reception is proposed. The first stage extracts the desired signal along each individual direction of the coherent components, and the second stage then combines the outputs of the first stage coherently. Numerical examples show that the proposed beamformer can achieve optimum performance. It is noteworthy that for coping with the performance degradation due to sample covariance errors, DOA estimation errors and other array imperfections, we can use the eigenstructure of the covariance matrix that constrains the weight vector along individual direction in the signal subspace [6] to enhance the performance of the proposed approach.

\section{REFERENCES}

[1] VAN TREES, H.L.: Optimum Array Processing, John Wiley \& Sons, Inc. 2002

[2] GONEN, E. and MENDEL, J.M.: "Applications of cumulants to array processing-Part III: blind beamforming for coherent signals," IEEE Trans. Signal Processing, vol.45, no.9, pp.2252-2264, Sept. 1997

[3] WU, Q. and WONG, K.M.: "Blind adaptive beamforming for cyclostationary signals," IEEE Trans. Signal Processing, vol.44, no.11, pp.2757-2767, Nov. 1996

This paper is a postprint of a paper submitted to and accepted for publication in Electronics Letters and is subject to Institution of Engineering and Technology Copyright.

The copy of record is available at IET Digital Library. 
[4] YEH, C.C., and WANG, W.D.: "Coherent interference suppression by an antenna array of arbitrary geometry," IEEE Trans. Antennas Propag., vol.37, no.10, pp.1317-1322, Oct. 1989

[5] LEE, J.H. and HSU, T.F.: "Adaptive beamforming with multiple-beam constraints in the presence of coherent jammers," Signal Processing, vol.80, no.11, pp.2475-2480, Nov.2000

[6] YU, Y.L. and YEH, C.C.: "Generalized eigenspace-based beamformers," IEEE Trans. Signal Processing, vol.43, no.11, pp.2453-2461, Nov. 1995

This paper is a postprint of a paper submitted to and accepted for publication in Electronics Letters and is subject to Institution of Engineering and Technology Copyright.

The copy of record is available at IET Digital Library. 